\title{
CASES DEMONSTRATED AT THE F.R.C.S. CLASS.
}

\section{Case of Ulnar Nerve Palsy.}

Clinical Notes.-The patient, a house decorator by trade, put his arm through a window on June 30, 1932, and sustained an incised wound just above the inner side of his left elbow. He was admitted to St. Mary's Hospital with signs of a complete ulnar nerve lesion and a primary suture of the divided nerve was performed.

On examination (October 4) a very typical picture of an ulnar palsy is present. All the muscles supplied by the nerve are paralysed, including the flexor carpi ulnaris, and there is loss of protopathic, epicritic and muscular sensation corresponding exactly with the distribution of the ulnar nerve. The paralysis of the interossis, the thumb adductors and the hypothenar muscles is well shown. There is little wasting and the trophic condition is extremely good, both being attributable to physical treatment.

On palpation and percussion along the course of the nerve a tender point was discoverable, $12 \mathrm{~cm}$. below the lesion, at and below which tingling could be produced in the cutaneous distribution (Tinel's sign). This showed that regeneration was proceeding satisfactorily, at a rate of nearly half an inch a week, which compares favourably with the normal rate of $\mathrm{I} \mathrm{mm}$. a day.

Discussion.-The diagnosis was readily made by practically all candidates, but several failed to elicit Tinel's sign which was a special feature of the case. Many candidates were inaccurate in the prognosis and failed to lay sufficient emphasis on the factor of occupation. It is the patient's occupation which governs the prognosis of ulnar nerve lesions. To a surgeon or pianist the hand would be almost useless as the more delicate and complex movements would never return in their original perfection. A labourer, on the other hand, would hardly notice the lesion, even if recovery were poor. Case presented by A. J. Cokkinis, F.R.C.S.

\section{A Case of Syringomyelic Arthropathy of the Elbow Joint.}

This case was one of those shown to the F.R.C.S. class.

The patient was a woman, aged 46 , who had noticed a painless swelling of the right elbow-joint for five years.

Examination showed a generalized enlargement of the right elbow with some periarticular thickening. There was slight limitation of flexion and extension but marked lateral mobility was present allowing free and painless circumduction of the forearm. There was no evidence of fluid in the joint.

Loss of thermal appreciation and of pain was present below the elbow, but there was also some diminution of tactile sensibility over the whole of the arm.

There was slight wasting of the thenar and hypothenar eminences, but no suggestion of "claw hand."

Examination of the fingers showed that the terminal phalanx of the thumb was missing (it had been amputated three years previously for a painless whitlow) the nails were ridged and distorted, and there was a circular scarred area on the second finger where the patient had scalded herself a short time before without noticing any pain.

The pupils were small but equal and reacted to light. There was no enophthalmos or pseudoptosis.

The knee-jerks were slightly exaggerated. There was no scoliosis. The Wassermann reaction was negative. 
$\mathrm{X}$-rays of the elbow showed disorganization of the joint, the actual articular changes being a mixture of the atrophic and hypertrophic types in that the head of the radius had completely disappeared, while the extremities of the humerus and ulna showed hypertrophic changes with the formation of irregular bony masses.

Most of the candidates diagnosed the condition as a neuropathic arthritis but attributed this to tabes and not to syringomyelia.

My main criticism was that the examination of the case was, in several instances, hurried and rather perfunctory. They confined their attention to the elbow-joint and omitted to observe the clue furnished by the condition of the hand.

Several overlooked the fact that the terminal phalanx of the thumb was missing and that the nails were abnormal in appearance. Moreover, though the majority elicited the knee-jerks and tested the reaction of the pupils to light, few looked to see if the pupils were equal and if a lateral curvature of the spine was present. (Inequality of the pupils-that on the effected side being smaller-and scoliosis are signs which are usually present in syringomyelia.)

In this case true dissociated anæsthesia was not present as in addition to the loss of pain and of thermal dissemination there was in addition some loss of tactile sensation.

The latter finding being uncommon.

The slightly exaggerated knee-jerks were due to pyramidal involvement.

Case presented by T. Meyrick Thomas, F.R.C.S.

\section{Epithelioma of Tongue Treated by Radium.}

B. T., male, aged 56 .

History.-Four months' history of lump on inferior surface of tongue, which increased in size and ulcerated.

General condition good.

Local condition-irregular ulcer 3 by $3.5 \mathrm{~cm}$. on inferior surface of tongue. fixation. All teeth extracted.

Edges of ulcer raised and hard-centre covered hy slough.

No glands palpable.

Wassermann reaction-positive.

No section taken.

The appearance on admission to the Cancer Hospital was that of a typical epithelioma. A coloured drawing of the original condition was shown to candidates after the patient had been examined by them.

When exhibited on September 29, all traces of growth had disappeared, but the front of the tongue and floor of the mouth showed a typical radium reaction. The patient stated that bilateral submaxillary swellings came and went within a few hours and that these were associated with the taking of food.

Several candidates were not acquainted with the appearance of a typical radium reaction, and suggested that the condition was a glossitis due to syphilis. It was impossible to reconcile the submaxillary swellings with such a supposition. There was little or no indication that the neck had received any irradiation. The skin over the area treated was rather more smooth than normal, but there was no skin reaction although the dosage to the area had amounted to nearly thirty gramme hours. 
Treatment.-August 19,1932 : Radium needles inserted into tongue.

Dose-I5 (I) mg. I34 hours-2006 mg. el. hrs. Filter : $0.5 \mathrm{~mm}$. Pt.

September 16 : Bomb treatment begun to submental region.

October I : Bomb treatment completed to submental region.

Dose-30 grm. hours. Distance : $5 \mathrm{~cm}$. Filter : $\mathrm{I} \mathrm{mm}$. Pt. Size of field : $5 \mathrm{~cm}$. diameter. Content of Bomb I grm.

Case presented by Percival F. Cole, F.R.C.S.

\section{A Lump in the Groin.}

History.-A labourer, aged 32, was shown, complaining of a swelling in the left groin. Sixteen years previously, about ten days after a kick in the left hip region, this had been operated upon while he was feverish and delirious, and tubes left in. For many years now he had been doing heavy work and walking painlessly, though with a slight limp. He had now been " run down" for weeks, "off his food," and had lost a stone in weight in one month. Two weeks ago he had experienced a sudden pain in the left groin, first felt on walking, and a lump developed there, which at first "got smaller during the night," but soon "became fixed in size." There had been no abdominal pain, nausea, cough, or pain on walking, but the bowels had been constipated.

Examination.-The patient looks well. The left lower limb is wasted and truly shortened, and the left hip, which is flexed and adducted, allows no abduction, and only limited rotation and flexion, which are painless. There is a vertical irregular scar, about 6 in. long, over the left great trochanter.

Below the inner end of the left Poupart's ligament is a smooth, rounded, tender, soft, solid, irreducible lump, having no expansile impulse on coughing, movable in all directions, and having a small upward and outward projection. The skin, here pink, can be lifted off the swelling except at its centre.

Above the outer part of Poupart's ligament on the same side is a firm, tender swelling, about $\mathrm{I}$ in. by 2 in. in diameter, with its long axis parallel to the ligament, and fixed to the posterior abdominal wall. This swelling is generally smooth, but has small irregularities on its surface. There is no clinical evidence, nor is there any history, of recent sepsis in the areas of lymphatic drainage of the left iliac and inguinal glands.

Discussion.-The history and the position and consistency of the lower swelling might at first sight suggest an irreducible femoral epiplocele, but this was put out of court by the facts that it was impossible to pick the skin off the centre of the lump, that this had no impulse on coughing, and that it was movable in all directions.

About half the candidates failed to discover the swelling above Poupart's ligament. With any swelling below this ligament, the region above should always be carefully examined. An apparent femoral hernia may be a psoas abscess. About one quarter of the candidates said that the lower swelling fluctuated, most that it did not, while the adjective fluctuant was also used. A soft solid body, such as an enlarged prostate, may be described as elastic, but it does not fluctuate. Fluctuation is either present or absent. If one is doubtful, the swelling should be termed elastic. Such a swelling usually proves to be a soft solid, and not liquid. Fluctuant is a bad term, and should be avoided as significant of mental fluctuation.

With regard to further investigation, while most candidates rightly desired the help of radiography, simpler aids were neglected. Thus, in spite of local signs of inflamma- 
tion, only one candidate inquired whether pyrexia had been present, and no one asked for a leucocyte count.

One observer diagnosed the lower swelling as a sebaceous cyst. This was a bad shot, because of the short history and absence of fluctuation, and because it ignored the swelling above Poupart's ligament.

Some diagnosed a neoplasm in the left iliac fossa with secondarily enlarged glands. This was not unreasonable; but against it were the tenderness and general smoothness of the swelling above Poupart's ligament, the signs of inflammation in the swelling below, the X-ray appearances, the short history, pyrexia (to $100^{\circ}$ on the last three days, the pulse-rate being roo), and the generally healthy aspect of the patient. Had the pyrexia been associated with a rapidly growing sarcoma, the patient would almost certainly have looked ill, and the radiograms given definite indication. These merely showed gross deformity and flattening of the head of the left femur, with "fluffiness" of the joint.

Most candidates suggested that the swellings were enlarged glands, though some, forgetting the presence of glands along the deep circumflex iliac vessels, doubted the possibility of the upper swelling being glandular.

The case, admittedly difficult, was shown as a means of testing methods of examination, a knowledge of which is more important than the ability to make lucky diagnostic shots in the dark. The provisional diagnosis was subacute left iliac and inguinal lymphadenitis, presumably secondary to a smouldering osteitis of the left ilium-a mild recrudescence from his old acute inflammatory attack.

At operation three days later, on September 30 , by Mr. Simmonds (to whom and to Mr. Addison I am indebted for permission to show, and report on, this patient), a veryo little yellow pus at once escaped, and the lump below Poupart's ligament was excised.? This consisted of a large lymph gland with broken-down tissue on its inner side. Nothing could be aspirated from the swelling above Poupart's ligament.

A non-hæmolytic streptococcus was grown from the gland, in which microscopy showed multiple areas of suppuration, and also many plasma cells. The Wassermann reaction was negative. The swelling above Poupart's ligament rapidly subsided after operation. Since the radiograms showed no definite sequestrum, one is left in doubt as to the source of sepsis leading to the development of the swellings, which were undoubtedly subacutely inflamed lymph glands.

Case presented by Alex. E. Roche, F.R.C.S.

\section{Comments on a Case of Multiple Gummata of the Tongue.}

History.-The patient, Mrs. R. D., aged 36, was referred to me in September, I931, after having had the majority of her teeth extracted for pyorrhœa and septic stumps. She then complained of a solitary, oval lump, about the size of a marble, in the right half of her tongue. She could not explain how the lump arose, but was inclined to attribute it to undue activities on the part of the dentist.

I made a provisional diagnosis of "fibroma or organizing hæmatoma," and discharged her with the request that she would come and see me again in the course of a month or two. I heard no more until three weeks ago, October 5, 1932, when she was again referred to me by her doctor with a diagnosis of "adeno-carcinoma of the tongue with secondary involvement of the glands in the neck"! 
Examination.-The patient looked very pale, sallow and anæmic. The gums had soundly healed over where the teeth had been extracted. The tongue was enormously enlarged, and contained eight tumours, scattered over its whole area. The lumps were mostly circular or oval, very hard in consistency, and were located in the actual substance of the tongue, bulging the anterior surface into multiple irregular excrescences. The lumps varied in size, the smallest being about $\frac{1}{4}$ in. in diameter, and the largest about $\frac{1}{2}$ in. The surface of the tongue was denuded of epithelium, and presented numerous cracks and fissures, in the depths of which were pent-up, decomposing epithelium and debris. The tumours did not extend to the under surface of the tongue, or cause any deformity in this region. The organ, although indurated, was freely mobile in all directions, and could be protruded well beyond the margin of the lips. The condition was quite painless, even to firm pressure, and no fluctuation could be made out over any of the tumours ; in fact, they were all of a stony hard consistency. In each submaxillary triangle masses of large, adherent inflammatory glands could be identified. The pupils reacted to light and accommodation, knee-jerks were present, and there were no other evidences of syphilitic trouble. A complete blood-count showed a marked secondary anæmia, and a Wassermann reaction was positive to the extent of +++ .

For a fortnight before attending the F.R.C.S. Class at the London Temperance Hospital she was given a brisk course of anti-syphilitic treatment.

Comments. - In view of the extreme rarity of multiple gummata of the tongue I thought that this would be an excellent case for the Fellowship Course. Over 50 per cent. of the candidates made a wrong diagnosis, and an enumeration of some of the diversities of their opinions is here given :-

(I) Adeno-carcinoma.

(5) Diffuse epithelioma.

(2) Lymphangioma.

(6) Mucous patches, and

(3) Angiomata.

(7) Multiple papillomata.

(4) Multiple fibromata.

It is inconceivable how some of these candidates could have arrived at such a diagnosis ; for instance, an adeno-carcinoma of the tongue (which I have never seen) must be a pathological curiosity, and how a diagnosis of multiple papillomata could have been made in the case of a tongue with a glazed surface without epithelium is incomprehensible.

The majority of mistakes are made through imperfect or casual examinations, or in some instances through fundamental lack of the knowledge of anatomy and pathology.

Case presented by Rodney H. Maingot, F.R.C.S.

\section{Swellings of the Neck.}

I showed four cases in the evening of October II. Three of these presented swellings in the neck, and as many mistakes were made by the men who saw these cases, largely because of the "leg pulling" complex of the advanced student, the fear of making an obvious diagnosis when presented with an obvious clinical picture, I should like to discuss the three together.

The first was a boy of about 8 , with a median sinus between the hyoid and thyroid cartilage. The edges of the sinus were red, the centre puckered, and a thin yellowish 
discharge came from its orifice. The deeper parts of the sinus were firmly attached to the fascia in the mid-line, and when he swallowed, the whole scar was drawn up. He told us that a lump had appeared for the first time three months before and had burst spontaneously.

Here the diagnosis was clearly one of a fistula of the thyroglossal duct. Many men, however, impressed by the fact that nothing wrong had been noticed in the neck till a few months earlier, called the condition a breaking down tuberculous gland. It is very unusual to find one suppurating tuberculous gland with no other glands palpable anywhere in the neck, or to find a single tuberculous gland in the mid-line and none in the submaxillary or carotid triangles. It is so unusual to find a lymphatic gland of any sort in the mid.line of the neck between the hyoid and the cricoid, that it is almost safe to say that such glands only occur in the figures of advanced anatomical textbooks devoted to the lymphatic system. In any case, a tuberculous sinus is not drawn right up when the patient swallows.

A short history is by no means unusual in the case of swellings in the neck of congenital origin, probably explained by the fact that a previously impalpable cavity has become rapidly distended owing to infection of its contents or in its neigbourhood. The liability of cystic hygromata to infection is well known; inflammatory changes in remnants of the thyroglossal duct are only slightly less frequent. The median portion of the thyroid is developed from a tubular downgrowth from the pharynx, which remains patent through life in Amphioxus, but in man disappears, leaving only the foramen cæcum to indicate where it lay. Parts of this downgrowth may remain as a tube, or they may become ectopic thyroid tissue ; in cases coming to operation we nearly always find a tubular cavity lined with cubical or columnar epithelium, and extending aco considerable distance upwards beyond the palpable limits of the swelling. For this? reason the operation for their removal should always include resection of about $\frac{1}{3}$ in. of the centre of the body of the hyoid. The theoretical relationship of this duct to the hyoid is open to argument; in practice it may be found passing in front of, behind, or through the bone. When the swelling in the neck has been isolated, and the hyoid cut across on each side of it, traction will bring the upward extension into view as it passes between the muscles of the tongue in the mid-line, and complete removal of the whole tract is comparatively easy.

The second case was a woman of about 60 , with a lump on the right side of the neck. The lump was oval, about 4 by 3 in. across, and extended from the posterior to the anterior triangle, being crossed rather in front of its middle by the sternomastoid. The skin and superficial fascia could be moved freely over it, but it could only be moved to a very small extent over the deeper structures. When the patient swallowed, the lump appeared to change its shape owing to the contraction of the over-lying sternomastoid muscle, but it did not move upwards as a whole. It was very soft, gave fluctuation with great readiness, and transilluminated brightly, a sign, however, that was easily missed in the well-lit Out-patient Department of the Temperance Hospital. It was painless and not tender. The patient had only noticed the swelling during the last three months, in which time it had grown appreciably. Apart from its appearance it caused her no inconvenience, and her general health always had been, and still remained, excellent.

The swelling was a lymphatic cyst, a diagnosis that was only made by about one in 
ten of the candidates who examined it. At operation the next day it shelled out with the greatest readiness, and was found to consist of a very thin wall, like that of a spermatocele, enclosing straw-coloured fluid. It separated readily from surrounding structures, but a few small lymphatic glands appeared to be incorporated in its coverings. What exactly is the nature of these lymphatic cysts arising comparatively rapidly in adults I am unable to say. This is the fourth I have removed; of the others two were in the axilla, and one in Scarpa's triangle. I am inclined to the view that they are cystic hygromata of congenital origin, which have been unnoticed while they remained small, and have become distended owing to inflammatory changes.

One reason for a wrong diagnosis here was incomplete examination. The laxity and free fluctuation put all the common swellings in the neck out of court, the only common swelling in the neck that does not develop tension as it enlarges and so becomes tight and elastic, is a cold abscess. This then was an unusual swelling, fluctuating, and therefore containing either fluid or gas. Without transillumination and percussion, the ordinary means of investigating its contents had not been tried. Air cysts in the neck are excessively rare, but the satisfaction that comes from their discovery in practice, and the kudos that arises from their identification at an examination, well repays the trouble of a flick with the finger.

Transillumination here excluded a cold abscess, and also those two conditions which were the favourite diagnoses of the candidates-branchial cyst and goitre. Branchial cysts, though congenital swellings, are usually seen in adults, but seldom as old as this lady; the commonest age is between 18 and 30. They increase in size steadily and slowly, so that a history of at least a year may be expected. They occupy an almost constant position, about the level of the upper border of the thyroid cartilage, and mostly in the anterior triangle, though extending to some extent deep to the sternomastoid. They are moderately tense, and they never transilluminate, being filled with putty-like material containing numerous cholesterin crystals. In theory cysts should also occur which arise from the deeper part of the branchial clefts, lined with cubical epithelium and filled with clear fluid; these, however, are never encountered clinically. Swellings connected with the thyroid are never translucent,' and except for the carcinomata which do not attain the size of the present tumour, move up with the larynx on swallowing; they do not enter the posterior triangle except in the case of the fœetal adenomata of childhood, in which rounded lumps of the size and consistency of grapes may extend right across the neck, even those in the posterior triangle moving with the thyroid.

The third case was a woman with an adenoma about the size of a golf ball in the posterior part of the left lateral lobe of the thyroid, deep to the sternomastoid. Most of the candidates made the correct diagnosis, but about one in four called it a carcinoma because of its hardness. It is a commonplace that the thyroid adenomata that appear to be cystic are solid, and those that appear to be solid are cystic. Most adenomata undergo degeneration when they exceed an inch in diameter, and their walls then become thickened, containing dense fibrous tissue, cartilage or even calcareous plates. Such changes accounted for the hardness in the present case; but the lump not only moved freely on swallowing, but could be pushed up and down on the thyroid cartilage, whereas a carcinoma of such a size would have been firmly fixed and less definite in 
outline. This.lady also had a rounded swelling in the subcutaneous tissues of the back of the neck, for which the favourite label was a lipoma. It is only the subfascial lipomas $\stackrel{\bar{D}}{2}$ that are really smooth; those in the subcutaneous tissues always show some lobulation. Rounded elastic swellings in the subcutaneous layer and not attached to deeper $\stackrel{\vec{s}}{\vec{s}}$ structures always turn out to be sebaceous cysts, even if their attachment to the skin is? not demonstrable.

Cases presented by W. H. OgII.viE, F.R.C.S.

\title{
CALCIUM METABOLISM AND THE PARATHYROIDS.
}

\author{
BY W. LANGDON BROWN, M.A., M.D.CANTAB., F.R.C.P. \\ Regius Professor of Physic in the University of Cambridge.
}

THE discovery of the control over calcium metabolism exerted by the parathyroids and 3 by vitamin $D$ has led to a renewal of interest in the subject. Calcium had been regarded as a very inert substance, as it is deposited in the largest amounts in normal tissues with a sluggish metabolism, or in any dead tissue which is not infected, but it is so large or $\frac{A}{v}$ so situated that it cannot be absorbed. The replacement of elastic tissues by calcareous? material is a characteristic feature of growing old. But all the activities of calciumcannot be disposed of so summarily, for calcium salts are essential to the heart beat, and, indeed, if they were simply inert, no bad results would follow their removal from the diet, which we know is not the case.

For a comprehensive view of the recent additions to our knowledge on this subjegt we are indebted to the work of Donald Hunter, and in this brief résumé of the prese position I freely acknowledge that indebtedness.

In the first place, I should like to say a few words on the association between the thyroid gland and calcium metabolism.

In hyperthyroidism the output of calcium may be increased two to eightfold, and out of all proportion to the increase in the basal metabolic rate. In this condition, $\stackrel{\circ}{\vec{D}}$ much of the calcium is excreted by the bowel, and that without any excess of calcium in the blood. This increased output cannot be due to the thyroid stimulating the parathyroid, or the blood-calcium would be high. Yet like the parathyroid, the thyroid appears to mobilize the calcium from the bones-in less than half the cases, osteoporosis occurs-but it can be prevented by an adequate calcium diet. It is produced by progressive bone absorption by osteoclasts.

But it is naturally in connection with the parathyroids that the question of calcium $\delta$ metabolism has recently come to excite special interest. I want to contrast the effects of parathormone and vitamin D on calcium metabolism and then to apply this to certain clinical conditions.

Although the rôle of the red bone-marrow in the formation of red corpuscles has been known for over sixty years, and for more than half that time it has been recognized that the amount and distribution of blood-forming marrow was not fixed, but fluctuated according to the needs of the organism, it is only recently that we have known that the same applies to the calcified structures of the bone as well. In the skeleton we have a great calcium bank where deposits are continually being paid and withdrawals made.

Although from the earlier days of the experimental study of total removal of the thyrord, tetany was connected in many people's minds with the coincident removal of 